\title{
Novel synthetic amino acid copolymers that inhibit autoantigen-specific $T$ cell responses and suppress experimental autoimmune encephalomyelitis
}

\author{
Masha Fridkis-Hareli, ${ }^{1}$ Laura Santambrogio, ${ }^{2}$ Joel N.H. Stern, ${ }^{1}$ Lars Fugger, ${ }^{3}$ \\ Celia Brosnan, ${ }^{4}$ and Jack L. Strominger ${ }^{1,2}$ \\ ${ }^{1}$ Department of Molecular and Cellular Biology, Harvard University, Cambridge, Massachusetts, USA \\ ${ }^{2}$ Department of Cancer Immunology and AIDS, Dana-Farber Cancer Institute, Boston, Massachusetts, USA \\ ${ }^{3}$ Department of Clinical Immunology, Aarhus University Hospital, Skejby Sygehus, Aarhus N, Denmark \\ ${ }^{4}$ Department of Pathology, Albert Einstein College of Medicine, Bronx, New York, USA
}

Address correspondence to: Jack L. Strominger, Department of Molecular and Cellular Biology,

Harvard University, 7 Divinity Avenue, Cambridge, Massachusetts 02138, USA.

Phone: (617) 495-2733; Fax: (617) 496-8351; E-mail: jlstrom@fas.harvard.edu.

Received for publication March 5, 2002, and accepted in revised form May 14, 2002.

Copolymer 1 (Cop 1, Copaxone [Teva Marion Partners, Kansas City, Missouri, USA]), a random amino acid copolymer of tyrosine $(\mathrm{Y})$, glutamic acid $(\mathrm{E})$, alanine $(\mathrm{A})$, and lysine $(\mathrm{K})$, reduces the frequency of relapses by $30 \%$ in relapsing-remitting multiple sclerosis (MS) patients. In the present study, novel random four-amino acid copolymers, whose design was based on the nature of the anchor residues of the immunodominant epitope of myelin basic protein (MBP) 85-99 and of the binding pockets of MS-associated HLA-DR2 (DRB1*1501), have been synthesized by solid-phase chemistry. Poly (Y, F, A, K) (YFAK) inhibited binding of the biotinylated MBP 86-100 epitope to HLA-DR2 molecules more efficiently than did either unlabeled MBP 85-99 or any other copolymer including Cop 1. Moreover, YFAK and poly (F, A, K) (FAK) were much more effective than Cop 1 in inhibition of MBP 85-99-specific HLA-DR2-restricted $\mathrm{T}$ cell clones. Most importantly, these novel copolymers suppressed experimental autoimmune encephalomyelitis, induced in the susceptible $\mathrm{SJL} / \mathrm{J}\left(\mathrm{H}-2^{\mathrm{s}}\right)$ strain of mice with the encephalitogenic epitope PLP 139-151, more efficiently than did Cop 1 . Thus, random synthetic copolymers designed according to the binding motif of the human immunodominant epitope MBP 85-99 and the binding pockets of HLA-DR2 might be more beneficial than Cop 1 in treatment of MS.

J. Clin. Invest. 109:1635-1643 (2002). doi:10.1172/JCI200215402.

\section{Introduction}

Multiple sclerosis (MS) is an inflammatory disease of the CNS affecting $0.1 \%$ of the population and is associated in northern European caucasoid MS patients with the HLADR2 (DRB1*1501) haplotype (1-3). The animal model of MS, experimental autoimmune encephalomyelitis (EAE), is a T cell-mediated autoimmune disease that can be induced by subcutaneous injection of peptides derived from myelin components such as myelin basic protein (MBP) (4-6), proteolipid protein (PLP) $(7,8)$, or myelin oligodendrocyte glycoprotein (MOG) (9). In the course of EAE, autoreactive $\mathrm{CD}^{+} \mathrm{T}$ cells recognize self-antigens presented by murine class II MHC molecules (e.g., $\mathrm{H}-2^{\mathrm{s}}$ ), ultimately leading to pathological changes that can be monitored as clinical signs of disease. EAE provides a well-studied system for testing the efficacy of therapeutic compounds to suppress the disease. These have included treatment with cytokines $(10,11)$, peptide antigens that induce anergy (12), oral tolerance (13-15), or altered peptide ligands (16-19).

Copolymer 1 (Cop 1 ) is a random amino acid copolymer of alanine (A), lysine (K), glutamic acid (E), and tyrosine $(\mathrm{Y})$ in a molar ratio of approximately 5:3:1.5:1 synthesized in solution using $N$-carboxyamino acid anhydrides (20). Initially, this and other related copolymers were used to define the genetic basis of immune responsiveness, in genes now known as class II MHC genes $(21,22)$. Later, Cop 1 was found to be effective both in suppression of EAE $(20,23-25)$ and in the treatment of relapsing forms of MS (26-28). Its activity was suggested to involve, as a first step, binding to class II MHC proteins on the surface of the antigenpresenting cell (APC) (29). Its effectiveness may be due to competition with myelin antigens (MBP, PLP, and MOG) for activation of specific effector $T$ cells recognizing peptide epitopes derived from these proteins (29-31), and/or to induction of antigen-specific regulatory T cells $(25,32,33)$. After completion of phase 3 clinical trials, Cop 1 was approved as a therapy for MS, and it is currently in wide use. However, it reduces the relapse rate by only about $30 \%$ and is certainly not curative for the disease. Moreover, the relationship of therapeutic effectiveness to the HLA haplotypes of MS patients, and particularly to HLA-DR2 (DRB1*1501), to which the disease is linked, has never been studied. Similarly, the ability of Cop 1 to inhibit HLADR2-restricted $T$ cell clones derived from MS patients has only recently been reported (34). 
Thus, further investigation of the mechanisms involved, as well as examination of additional copolymers of this type, could potentially result in improved therapeutic reagents. Virtually all of the large variety of copolymers found in the random mixture of poly $(\mathrm{Y}, \mathrm{E}$, A, K) (YEAK) bound to purified human HLA-DR1, -DR2, and -DR4 molecules, showing that Cop 1 did indeed bind to purified class II MHC proteins (35). It also competed for binding of MBP 85-99 to HLA-DR2 (DRB1*1501) and inhibited responses of DR2-restricted T cells to MBP 85-99 $(34,35)$. The binding and inhibition of $\mathrm{T}$ cell clones occurred in spite of the fact that the $\mathrm{P} 1$ pocket in DR2 is too small to accommodate the hydrophobic residue $Y$ (due to the presence of $\beta 86 \mathrm{Val}$ rather than $\beta 86 \mathrm{Gly})$. However, at high concentration $Y$ may be "forced" into this pocket (36).

Thus, the combination of amino acids $\mathrm{Y}, \mathrm{E}, \mathrm{A}$, and $\mathrm{K}$ as in Cop 1 might not be the best for the potent inhibitory activity of this compound. Therefore, copolymers that took account of the binding motifs of the autoantigenic peptide derived from MBP (MBP 85-99) might be better therapeutic agents than Cop 1. In the present study, several random four-amino acid copolymers - 14-, 35-, and 50-mers with different ratios of amino acids - have been synthesized by the solid-phase method with particular reference to the anchor residues of MBP 85-99 bound to HLA-DR2 (DRB1*1501) (37, 38 ) in an attempt to improve the effectiveness of the copolymers. Biochemical analysis and effects of the new copolymers on autoantigen-specific $\mathrm{T}$ cell responses in MS and on disease progression in the animal model of MS (EAE) are presented here.

\section{Methods}

Copolymers, peptides, and proteins. YEAK, VEAK, and FEAK in molar ratios approximating those found in Cop 1 (1:1.5:5:3) were synthesized by the solid-phase method as 14-, 35-, and 50-mers (Mimotopes, Clayton, Australia), by using Fmoc amino acids mixed in the desired ratios at each cycle. YFAK, molar ratio 0.2:0.8:5:3, YFAK, molar ratio 0.8:0.2:5:3, YFAK, molar ratio $0.5: 0.5: 5: 3$, and FAK, molar ratio $1: 5: 3$, were synthesized similarly. Three different preparations of the novel copolymers were employed with identical results. Cop 1 (glatiramer acetate, Copaxone [Teva Marion Partners, Kansas City, Missouri, USA]) was obtained from the pharmacy and kindly provided by H.L. Weiner (Center for Neurologic Diseases, Brigham and Women's Hospital, Boston, Massachusetts, USA). Peptides were synthesized on an Applied Biosystems Peptide Synthesizer (Framingham, Massachusetts, USA) and purified by reverse-phase HPLC. Peptide sequences were: MBP 85-99, ENPVVHFFKNIVTPR, molecular weight 1795; PLP 40-60, TGTEKLIETYFSKNYQDYEYL, molecular weight 2603; and PLP 139151, HSLGKWLGHPDKF, molecular weight 1520; either unlabeled or with biotin linked to the N-terminus by the spacer SGSG and free acid at the C-terminus. MBP 86-100, rather than MBP 85-99, was used for biotinylation because of ease of synthesis. Soluble HLA-DR2 molecules were purified from Drosophila S2 cells as described (39).

Peptide binding to class II MHC proteins. Microtiter immunoassay 96-well plates (FALCON Pro-Bind; Becton Dickinson and Co., Lincoln Park, New Jersey, USA) were coated with $1 \mu \mathrm{g} /$ well affinity-purified LB3.1 mAb's in $100 \mu \mathrm{l} \mathrm{PBS}$ for 18 hours at $4^{\circ} \mathrm{C}$, as previously described (35). The wells were then blocked with TBS $(137 \mathrm{mM}$ sodium chloride, $25 \mathrm{mM}$ Tris [pH 8.0], $2.7 \mathrm{mM}$ potassium chloride) $/ 3 \% \mathrm{BSA}$ for 1 hour at $37^{\circ} \mathrm{C}$ and washed three times with TTBS (TBS plus $0.05 \%$ Tween-20). Biotinylated MBP 86-100 (final concentration $0.13 \mu \mathrm{M}$ ) in $50 \mu \mathrm{l}$ of PBS was co-incubated with unlabeled inhibitors (random copolymers or MBP 85-99), and with HLA-DR2 molecules, for 40 hours at $37^{\circ} \mathrm{C}$ and subsequently transferred to the 96-well plates treated as described above. Following 1 hour of incubation at $37^{\circ} \mathrm{C}$, plates were washed three times with TTBS and incubated with $100 \mu \mathrm{l}$ of streptavidin-conjugated alkaline phosphatase (diluted 1:3,000 in TTBS; Bio-Rad Laboratories Inc., Richmond, California, USA) for 1 hour at $37^{\circ} \mathrm{C}$, followed by addition of $100 \mu \mathrm{l} p$-nitrophenyl phosphate in triethanolamine buffer (Bio-Rad Laboratories Inc.). The absorbance at $410 \mathrm{~nm}$ was monitored by a microplate reader (model MR4000; Dynatech Laboratories, Chantilly, Virginia, USA).

Antigen presentation assays. HLA-DR2-restricted $\mathrm{T}$ cells were MBP 84-102-specific (or, more exactly, MBP 8599-specific) transfectants using $\mathrm{T}$ cell receptors (TCRs) obtained from patients with relapsing-remitting MS carrying DR2 [clone 8073, from patient Ob (DRB1*1501), and clone Hy1B, from patient Hy (DRB1*1602)], transfected into murine BW 58 TCR $\alpha^{-} / \beta^{-}$cells. MBP 84102-specific (2E12) and PLP 40-60-specific (106A) hybridomas from HLA-DR2 transgenic mice (6) were also used. Mouse T cell hybridomas were PLP 139-151-spe-

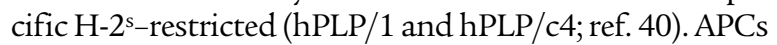
were L466 [L cells transfected with HLA-DR2b (DRB1*1501)], L416 [L cells transfected with HLA-DR2a (DRB5*0101)], MGAR (Epstein-Barr virus-transformed B cells homozygous for DRB1*1501), and splenocytes from $\mathrm{SJL} / \mathrm{J}\left(\mathrm{H}-2^{\mathrm{s}}\right)$ mice. $\mathrm{T}$ cell stimulation experiments were performed in a total volume of $200 \mu \mathrm{l}$ in 96-well microtiter plates. Irradiated (30 Gy) APCs $\left(2.5 \times 10^{4}\right.$ per well) were co-incubated with MBP 85-99, PLP 40-60, or PLP 139-151 and the random copolymers at concentrations indicated in Results, for 2 hours at $37^{\circ} \mathrm{C}$; then $\mathrm{T}$ cells $\left(5 \times 10^{4}\right.$ per well) were added and incubated for 24 hours at $37^{\circ} \mathrm{C}$. Supernatants $(30 \mu \mathrm{l})$ were taken and incubated with IL-2-dependent cytotoxic T lymphoma line cells $\left(5 \times 10^{4}\right.$ per well) for 12 hours, followed by labeling with ${ }^{3} \mathrm{H}$-thymidine $(1 \mu \mathrm{Ci}$ per well) for 12 hours. Plates were harvested and the radioactivity was monitored using a 1450 MicroBeta Plus liquid scintillation counter (Perkin Elmer Wallac Inc., Gaithersburg, Maryland, USA). Mice. SJL/J (H-2s) female mice (8-12 weeks of age) were purchased from The Jackson Laboratory (Bar Harbor, Maine, USA) and maintained in the animal 
facility at Harvard University according to the Guidelines of the Committee on Animals of Harvard University and the Committee on Care and Use of Laboratory Animal Resources, National Research Council (Department of Health and Human Services Publication 85-23, revised 1987).

Induction and suppression of EAE. Mice were injected subcutaneously in both the base of the tail and the nape of the neck with either mouse whole spinal cord homogenate (WSCH, $500 \mu \mathrm{g} /$ mouse) or PLP 139-151 peptide $(50 \mu \mathrm{g} /$ mouse) together with $400 \mu \mathrm{g}$ Mycobacterium tuberculosis H37Ra (BD Diagnostic Systems, Sparks, Maryland, USA) in an emulsion containing equal parts of PBS and CFA (Sigma-Aldrich). Pertussis toxin (List Biological Laboratories Inc., Campbell, California, USA; $200 \mathrm{ng}$ ) was injected intravenously into the tail 1 day after immunization. Mice were scored daily for clinical signs of EAE on a scale from 1 to 5 according to the severity of the disease as previously described $(40,41)$. For suppression of EAE, different copolymers $(500 \mu \mathrm{g} /$ mouse $)$ were injected together with the encephalitogenic emulsion as described above. Mice were evaluated in a blinded fashion. Maximum clinical score and mean day of onset were calculated as described in ref. 40.

Proliferation by lymph node primary cultures. Mice were immunized subcutaneously together with different copolymers emulsified in CFA, as described above, except that no pertussis toxin was administered intravenously. Lymph nodes and spleens were taken 13-16 days after immunization. Irradiated splenocytes $(12 \mathrm{~Gy}$, $5 \times 10^{5}$ cells per well) were incubated in 96-well roundbottom plates together with PLP 139-151 or copolymers at concentrations indicated in Results, for 2 hours at $37^{\circ} \mathrm{C}$, followed by addition of lymph node cells $\left(5 \times 10^{4}\right.$ cells per well) in DMEM/10\% FCS. For T cell proliferation, ${ }^{3} \mathrm{H}$-thymidine $(1 \mu \mathrm{Ci} /$ well $)$ was added to the duplicate set of cultures after 72 hours, and the plates were harvested and radioactivity monitored using a 1450 MicroBeta Plus liquid scintillation counter (Perkin Elmer Wallac Inc.) after 96 hours.

Neuropathology. For assessment of inflammation and demyelination, mice were perfused under anesthesia through the ascending aorta with $40 \mathrm{ml}$ of Trump's fixative (4\% paraformaldehyde, $1 \%$ glutaraldehyde in 0.1 $\mathrm{M}$ phosphate buffer, $\mathrm{pH}$ 7.4). Slices of the brain and spinal cord were postfixed in cold $1 \%$ osmium tetroxide for 1 hour, dehydrated through a graded series of ethanol, and embedded in epoxy resin. One-micrometer sections were stained with toluidine blue and examined by light microscopy.

\section{Results}

Preliminary synthesis and evaluation of novel copolymers $V E A K$ and FEAK. Initially, two new amino acid copolymers were synthesized to provide a better residue for binding in the P1 pocket of HLA-DR2 than is available in Cop 1. Since this P1 pocket is small in HLA-DR2 (DRB1*1501), V, the residue of MBP 85-99 found at P1, and $\mathrm{F}$, the largest hydrophobic amino acid that should fit in the P1 pocket of this HLA protein, were used instead of the Y present in Cop 1; i.e., VEAK and FEAK were synthesized. In addition, YEAK itself was synthesized by the solid-phase method to establish that this material has the same effectiveness as Cop 1 synthesized by solution chemistry. Various sizes of each copolymer, 14-mers, 35-mers and 50-mers, were synthesized. These copolymers were examined in three ways: (a) binding to "empty" HLA-DR2 (DRB1*1501) synthesized in a baculovirus system, (b) inhibition of the four MBP 85-99or PLP 40-60-specific HLA-DR2-restricted T cell clones, as well as the two PLP 139-151-specific H-2 ${ }^{\text {s-restricted }}$ $T$ cell hybridomas, and finally (c) ability to suppress EAE induced by either PLP 139-151 or WSCH in H-2s mice. The following results were obtained:

(a) In the binding experiment, VEAK and FEAK were less effective than Cop 1 or YEAK in competing for binding of biotinylated MBP 86-100 (data not shown);

(b) When tested as inhibitors of MBP 85-99- or PLP 40-60-induced stimulation of the four HLADR2-restricted $T$ cell clones or hybridomas, VEAK was substantially less effective than Cop 1 or YEAK in inhibition of proliferation, while FEAK was equivalent to Cop 1 using two of these clones and slightly less effective in the other two cases. Using the two PLP 139-151-specific $\mathrm{H}-2^{\mathrm{s}}$-restricted $\mathrm{T}$ cell hybridomas, again FEAK was approximately equivalent to Cop 1 or YEAK, while VEAK was half as effective. In both the binding and the inhibition of $\mathrm{T}$ cell proliferation experiments, the 50-mers of all of the copolymers used were much more effective than the 35 -mers or the 14-mers (data not shown).

Table 1

Suppression of EAE induced by WSCH in SJL/J mice by novel random copolymers VEAK and FEAK

\begin{tabular}{|c|c|c|c|c|c|}
\hline Copolymer & Incidence & Percent disease & Percent mortality & Maximal mean score & Mean day of onset \\
\hline- & $18 / 32$ & 56 & 3 & $2.2 \pm 1.2$ & $14.5 \pm 2.3$ \\
\hline Cop 1 & $1 / 14$ & 7 & 0 & 3.0 & 20.0 \\
\hline YEAK 50-mer & $2 / 16$ & 12 & 0 & 1.0 & 14.0 \\
\hline VEAK 50-mer & $8 / 16$ & 50 & 0 & $2.8 \pm 0.4$ & $18.3 \pm 7.4$ \\
\hline FEAK 50-mer & $0 / 16$ & 0 & 0 & - & - \\
\hline VEAK 35-mer & $3 / 9$ & 30 & 0 & $1.5 \pm 0.5$ & $19.0 \pm 3.0$ \\
\hline FEAK 35-mer & $0 / 10$ & 0 & 0 & - & - \\
\hline
\end{tabular}

See Methods for details. Three identical experiments with Cop 1 and the 50-mers were combined. The 35 -mers were used in only two of these. 


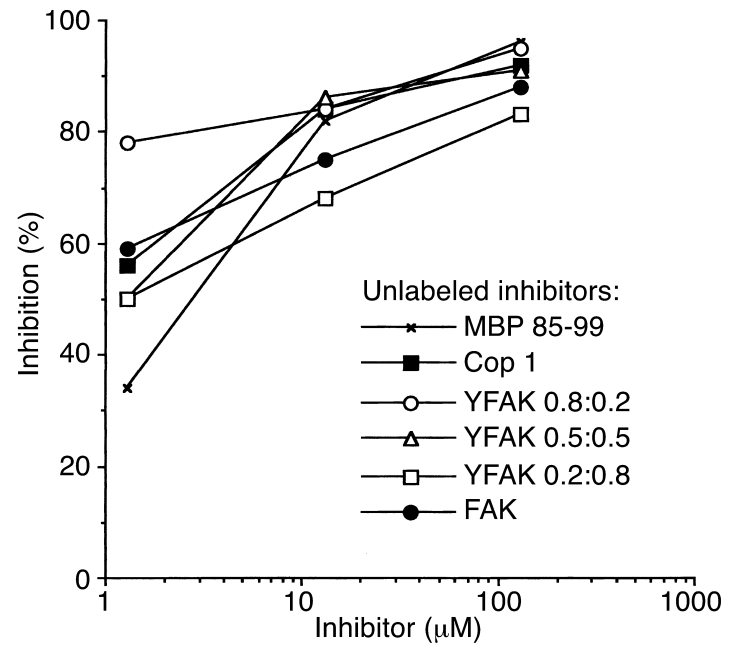

Figure 1

Inhibition of binding of biotinylated MBP 86-100 to HLA-DR2 molecules by different competitors. Recombinant water-soluble HLADR2 molecules were incubated with biotinylated MBP 86-100 (0.13 $\mu \mathrm{M})$ and the unlabeled random copolymers at a range of concentrations. All incubations were carried out in duplicate at $\mathrm{pH} 7.0$ for 40 hours at $37^{\circ} \mathrm{C}$. Results represent one of three independent experiments. Specific binding is expressed as percentage of inhibition using the formula: percentage of inhibition $=100 \%-[$ (absorbance at 410 $\mathrm{nm}$ with competitor - background)/absorbance without competitor - background) $\times 100$ ]. The signals at $410 \mathrm{~nm}$ without competitor were $0.8-1.0$, and the background was 0.1 .

(c) VEAK and Cop 1 were equally effective in partially reducing the severity of EAE induced by PLP 139-151 in $\mathrm{H}-2^{\mathrm{s}}$ mice, while FEAK completely prevented the appearance of disease, except for a few mice who developed a transient +1 score (limp tail). In immunohistology, brain sections of control animals and VEAK-treated animals showed substantial demyelination, while animals treated with Cop 1, YEAK, or FEAK were completely normal (data not shown). WSCH was also used to induce the disease, in which case a milder disease was produced, perhaps more comparable to MS. The mild disease persisted in some animals treated with either Cop 1 or YEAK and in an even larger number of animals treated with VEAK. However, no disease was detected in any animals treated with FEAK (Table 1).

Characterization of the additional novel random copolymers YFAK and FAK and their binding to HLA-DR2 molecules. Based on the initial results above, random four- and three- amino acid copolymers YFAK (at the molar Y/F ratios of 0.2:0.8, 0.5:0.5, and 0.8:0.2) and FAK were synthesized as 50-mers by the solid-phase method. F was substituted for E because: (a) E seemed unnecessary. Moreover, the $\mathrm{P} 1$ pocket of DRB1*1501 includes $\beta 86 \mathrm{Val}$, resulting in a small pocket that can accommodate $\mathrm{F}$ but for which $Y$ is too large to be accommodated (except at high peptide concentration; ref. 36). (b) The residue occurring at P4 in MBP 85-99 is F, although Y would provide a better fit (see Discussion). Amino acid analysis, reverse-phase HPLC separation, and microsequencing of the novel compounds showed that the solid-phase method yielded substances similar in amino acid composition, distribution, hydrophobicity, and size to Cop 1 that had been generated previously by solution chemistry (data not shown). To determine whether novel copolymers synthesized by the solid-phase method competed with the autoantigenic MS-associated epitope MBP 85-99 for binding to HLA-DR2, competitive binding assays were carried out with biotinylated MBP 86100 and unlabeled random copolymers (see Methods for details). All of the YFAK and FAK 50-mers were equally effective and equivalent to or better than Cop 1 in binding to HLA-DR2 (Figure 1).

Proliferative responses of MBP-specific T cells in the presence of the random copolymers. In a series of proliferation assays, the biological activity of the random copolymers was examined with several MBP 85-99-specific $T$ cell clones (see Methods). Three types of APC that express HLA-DR2 molecules were tested to determine which one presented the MBP 85-99 peptide most efficiently. Similar levels of proliferation were observed when this peptide was presented by MGAR [human lymphoblastoid B cell line that expresses DR2b (DRB1*1501)] or by L466 (murine L cell transfectant that expresses DR2b) (data not shown). When L416

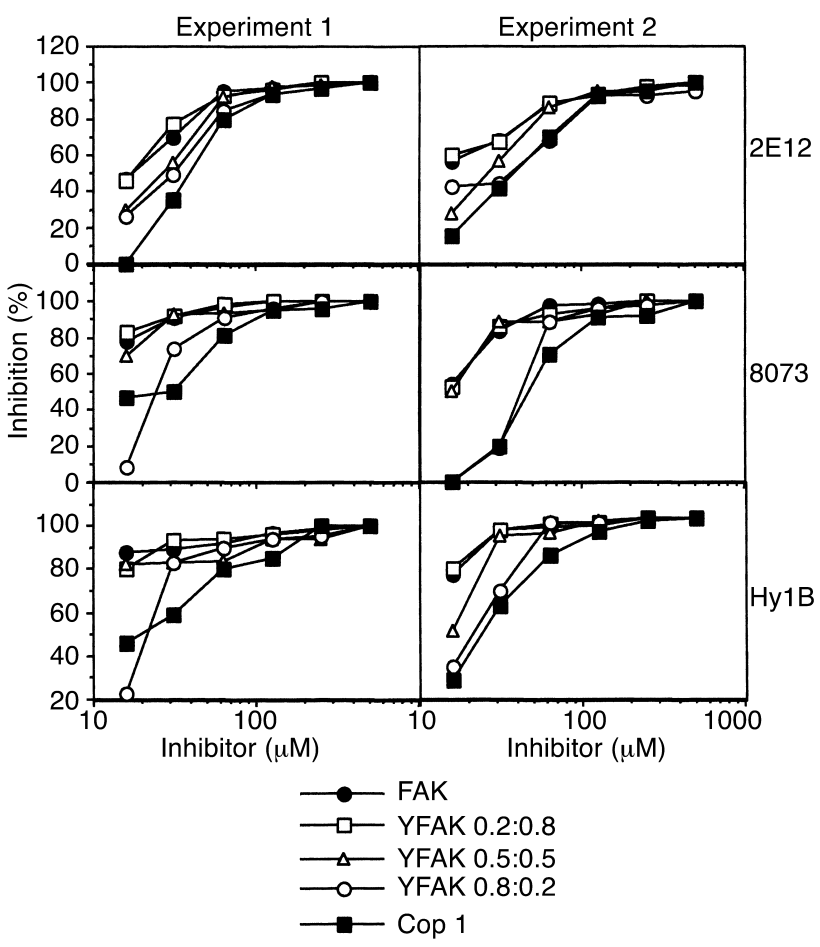

Figure 2

Inhibition of HLA-DR2-restricted MBP 84-102-specific T cells 2E12, 8073 , and $\mathrm{Hy} 1 \mathrm{~B}$ in the presence of the random copolymers. Irradiated MGAR cells were co-incubated in duplicate with the MBP 85-99 at the final concentration of $12.5 \mu \mathrm{M}$ and different concentrations of the random copolymers for 2 hours at $37^{\circ} \mathrm{C}$; then T cells were added and incubated for 24 hours at $37^{\circ} \mathrm{C}$. Supernatants $(30 \mu \mathrm{l})$ were incubated with IL-2-dependent cytotoxic T lymphoma line cells, followed by labeling with ${ }^{3} \mathrm{H}$-thymidine $(1 \mu \mathrm{Ci} /$ well $)$ for 12 hours. Other details were as described in Methods. 


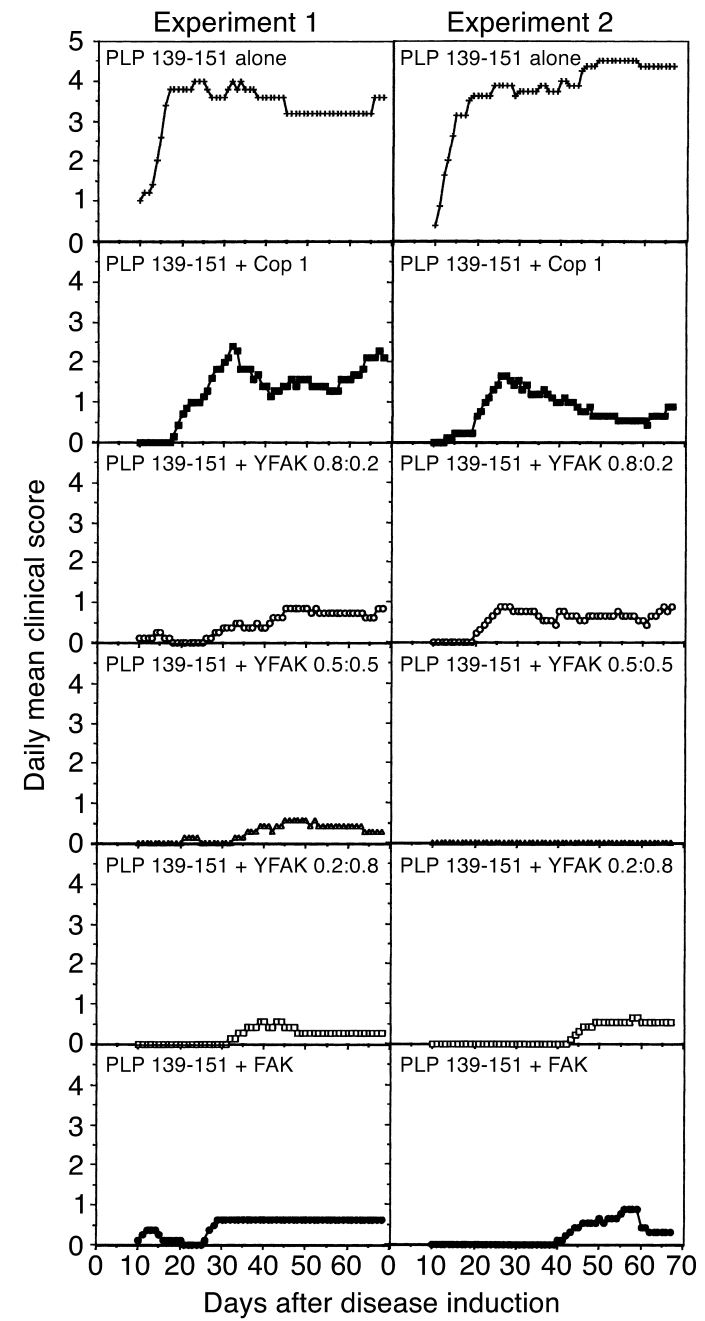

[L cell transfectant that expresses DR2a (DRB5*0101)] was used, no response was detected, confirming that all the $\mathrm{T}$ cell clones were restricted to the DR2b (DRB1*1501) allele (data not shown). Therefore, MGAR or L466 cells were subsequently used in the antigen presentation assays described below. The inhibition of proliferation of three different MBP 85-99-specific, DR2b-restricted T cell clones was examined in the presence of different copolymers. Two independent experiments are presented in Figure 2. For each MBP-specific HLA-DR2-restricted clone, YFAK 0.2:0.8, YFAK 0.5:0.5, and FAK were better inhibitors

\section{Figure 3}

Suppression of EAE induced with the PLP 139-151 epitope by different random copolymers. SJL/J mice were co-injected subcutaneously with $50 \mu \mathrm{g}$ of PLP 139-151 peptide and $500 \mu \mathrm{g}$ of the random copolymers FAK, YFAK (0.2:0.8), YFAK (0.5:0.5), YFAK (0.8:0.2), or Cop 1, or with PLP 139-151 alone. The progression of the disease was monitored for the appearance of clinical symptoms on a daily basis. Results represent the mean daily score. Five to nine mice per group were used in each of two independent identical experiments that were combined.

than YFAK 0.8:0.2 at lower concentrations of the inhibitors, with YFAK 0.2:0.8 being most effective. In any case, all these copolymers inhibited the MBP-specific HLA-DR2-restricted $T$ cell response more efficiently than did Cop 1.

Treatment of EAE induced by PLP 139-151 epitope. To find out whether the novel random copolymers affected chronic-relapsing EAE, in vivo experiments were carried out by immunizing SJL/J $\left(\mathrm{H}-2^{\mathrm{s}}\right)$ mice subcutaneously with $50 \mu \mathrm{g}$ of PLP 139-151 (the encephalitogenic epitope in the SJL/J strain) and $500 \mu \mathrm{g}$ copolymer. Following disease induction, mice were observed daily for the appearance of typical signs of EAE during a period of 70 days. Immunization with the PLP 139-151 epitope alone in CFA resulted in EAE with far more severe clinical signs compared with WSCH-induced EAE. Thus, all 13 mice developed severe EAE, with a mortality of $77 \%$. The first signs appeared around day 11, followed by subsequent fluctuation in disease attacks, with a maximal mean score of 4.6 (Figure 3; Table 2). Co-injection with the random copolymers differentially reduced the clinical signs of EAE. In the YFAK 0.2:0.8-treated group, only 2 out of 16 mice showed clinical signs of EAE (mortality, 6\%), with a delay in the first attack to around day 37 (maximal mean score, 0.6; Figure 3; Table 2). Similarly, in the YFAK 0.5:0.5-treated group, there was one sick mouse out of 16 (mortality, 0\%), with the first attack developing on day 33 . In contrast, 8 out of 17 mice treated with YFAK 0.8:0.2 developed EAE with no mortality. In this group, the maximal mean score (1.5) was higher than with the other YFAKs and the onset was earlier (day 27). Treatment with FAK resulted in 3 sick mice out of 17, with $12 \%$ mortality, maximal mean score of 0.9 , and the mean onset at day 25 (Figure 3; Table 2). However, when Cop 1 was coinjected with PLP 139-151, 12 out of 16 mice developed EAE, with the mean onset around day 22 and maximal mean score of 2.6. Figure 4 is a representative experiment

Table 2

Suppression of EAE induced by PLP 139-151 in SJL/J mice by novel random copolymers YFAK and FAK

\begin{tabular}{lccccc}
\hline Copolymer & Incidence & Percent disease & Percent mortality & Maximal mean score & Mean day of onset \\
- & $13 / 13$ & 100 & 77 & $4.6 \pm 0.8$ & $11.5 \pm 1.7$ \\
YFAK $0.2: 0.8$ & $2 / 16$ & 13 & 6 & $0.6 \pm 1.5$ & $37.5 \pm 7.7$ \\
YFAK 0.5:0.5 & $1 / 16$ & 6 & 0 & $0.2 \pm 1.0$ & $33.0 \pm 0$ \\
YFAK 0.8:0.2 & $8 / 17$ & 17 & 0 & $1.5 \pm 1.7$ & $26.6 \pm 10.7$ \\
FAK & $3 / 17$ & 75 & 6 & $2.9 \pm 1.8$ & $25.0 \pm 15.0$ \\
Cop 1 & $12 / 16$ & & & & 2.6 \\
\end{tabular}

See Methods for details. Three identical experiments were combined. 


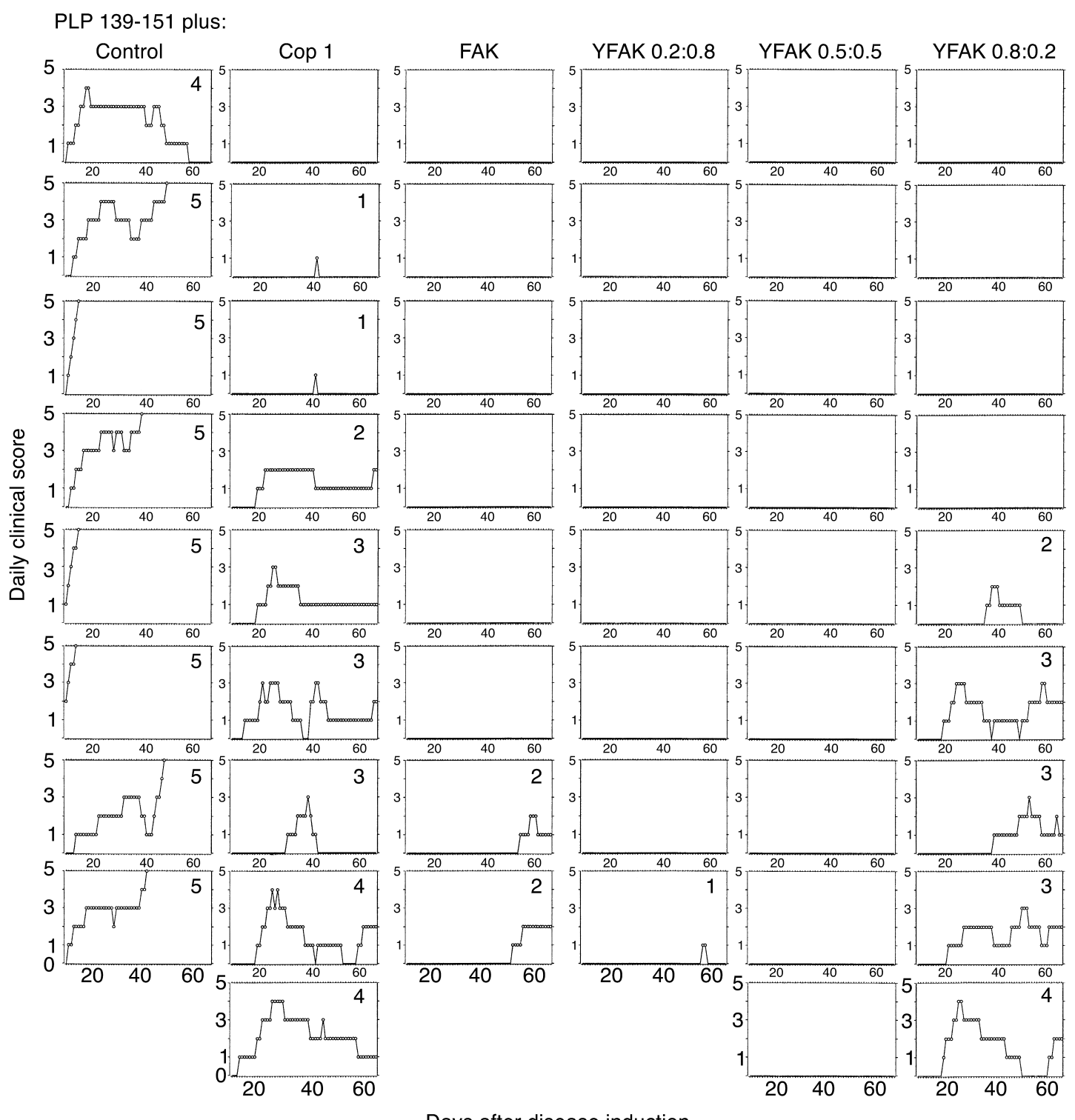

\section{Figure 4}

A representative experiment on suppression of EAE induced with the PLP 139-151 epitope, by different random copolymers. Each column represents a different treatment group as indicated on top, and each rectangle is an individual mouse in the group. Maximal disease score is shown for each mouse. Results represent the daily score of each individual mouse. Other details are as in the legend to Figure 3.

showing progression of the disease in each individual mouse out of the group treated with a specific copolymer during the 67-day period. In summary, random copolymers YFAK 0.2:0.8 or 0.5:0.5 and FAK were much more effective than Cop 1 in suppression of EAE. None of the mice treated with YFAK 0.2:0.8 or 0.5:0.5 developed significant disease.

\section{Discussion}

A major goal in the treatment of autoimmune diseases has been the development of antigen-specific immunomodulating therapies that interfere with the trimolecular interaction of the autoreactive TCR with the autoantigenic peptides presented by self MHC receptors at the surface of APCs. These immunotherapies of $\mathrm{T}$ cell-mediated autoimmune diseases have been successful in animal models with known target antigens $(10-19,42-45)$. One of the strategies, namely the use of altered peptide ligands, has been used both to treat $\operatorname{EAE}(12,17,19,46)$ and recently to treat MS $(47$, 48), although the results have been contradictory. Cop 1 , which was approved as a therapy for relapsing-remitting MS, was proposed to act as a promiscuous binder to class II MHC molecules $(29,35)$, as an antagonist of the TCR (49), and/or as an inducer of suppressor cells $(25,32,33)$. Although Cop 1 is currently in wide use, and a recent clinical study demonstrated its sustained efficacy in MS patients over a period of 6 years (50), it has only a modest effect on the course of disease, similar to other immunomodulatory drugs such as steroids or IFN- $\beta$. Development of novel compounds based on accumulated knowledge could thus result in improved 
therapeutic materials for MS and possibly for other autoimmune disorders.

Several novel random amino acid copolymers analyzed in this study were more potent in binding to HLA-DR2 molecules, inhibition of autoantigen-specific $T$ cells, and suppression of EAE than Cop 1 was. The design of these copolymers was mainly based on the residues of the immunodominant $T$ cell epitope MBP 85-99 interacting with the MS-associated HLA-DR2 (DRB1*1501) molecule. Interestingly, the size of the copolymers was crucial for activity, with the 50-mers being most efficient. In line with these data, previous study showed that 14-mer fragments of Cop 1, obtained by chymotrypsin digestion, bound poorly to the APC of different haplotypes (51), suggesting that longer polypeptides or multiple promiscuous epitopes are required for the higher affinity binding of this compound. Indeed, aggregation of class II MHC molecules on the surface of the APC upon Cop 1 binding was previously detected (52), suggesting that random polypeptides with an average of about 50-70 amino acids may be able to link adjacent class II molecules. Oligomerized T cell epitopes may cross-link HLADR molecules and thus enhance T cell responses (53).

The novel copolymers employed in the present study were optimized for binding to HLA-DR2. However, the murine model used for the in vivo studies was the SJL/J mouse. This strain lacks expression of I-E (the murine homologue of HLA-DR) and expresses only the I-As class II MHC molecule. However, this mouse model has previously been used to evaluate efficacy of Cop 1 prior to its introduction for the treatment of MS (31). That study assumed that data from this murine model were relevant to the treatment of the human disease, and it was therefore also employed in the present study. The structure of I-As (the homologue of human HLA-DQ protein) has never been resolved, and it remains for future work to determine whether Cop 1 and the novel copolymers described here bind to I-As in a manner similar to that of their binding to HLA-DR2. Earlier studies had indeed shown that Cop 1 binds promiscuously to living APCs of mouse origin (29), including SJL/J strain, suggesting that it interacts with the I-A $\mathrm{A}^{\mathrm{s}}$ molecules.

Among different random copolymers synthesized and examined in this study, YFAK and FEAK, but not VEAK, were most effective. Tissue samples from the lumbar cord of diseased mice injected with WSCH only or with WSCH and the 50-mer of the ineffective copolymer VEAK showed extensive submeningeal, perivascular, and parenchymal infiltration, as well as demyelination. In contrast, no infiltration or demyelination was detected in samples from mice who developed no signs of disease after treatment with the other copolymers (data not shown).

Importantly, the combination of $\mathrm{V}, \mathrm{E}, \mathrm{A}$, and $\mathrm{K}$, which appeared in some of the synthetic peptide 15 -mers designed according to binding motifs of the MBP 8599 epitope, resulted in a low-affinity binding to HLADR2 molecules and low levels of inhibition of HLADR2-restricted MBP 85-99-specific T cells (34). This observation is in contrast to the finding that, in the MBP 85-99/HLA-DR2 complex, $\mathrm{V}$ is the anchor residue at position 89 of the peptide interacting with $\beta 86 \mathrm{Val}$ in the $\mathrm{P} 1$ pocket of the HLA-DR2 protein (38). However, the substitution of F for V89 in MBP 85-99 resulted in a peptide that could bind to HLA-DR2 molecules (37) and may explain the higher inhibition by FEAK than by VEAK of the HLA-DR2-restricted MBP 85-99-specific $T$ cells. $\mathrm{F}$ can fit in the $\mathrm{P} 1$ pocket, but it also fits in the P4 pocket, as found at F92 in MBP 85-99. Thus, because FEAK binds in both pockets, it is a better binding copolymer. Although $Y$ would make a tighter fit in the $\mathrm{P} 4$ pocket, it is too large for the $\mathrm{P} 1$ pocket (and hence the lower efficacy of Cop 1, YEAK). The P4 pocket of DRB $1 * 1501$ is very large and can also accommodate the largest amino acid, W (37). The large size and hydrophobicity of this pocket are due to the unusual presence of the small $\beta 71 \mathrm{Ala}$, while virtually all other DR proteins have large charged residues at this position (e.g., $\mathrm{K}$ in DRB1*0401) (38). Thus, the combination of $\mathrm{F}$ at $\mathrm{P} 1$ and $\mathrm{Y}$ at $\mathrm{P} 4$ would stabilize the complex with DR2 (DRB1*1501) and may account for the enhanced efficacy of YFAKs, among which YFAK with $\mathrm{Y} / \mathrm{F}$ ratios of 0.5:0.5 and 0.2:0.8 appeared to be the most efficacious. It should be noted that YFAK 0.8:0.2 was the best inhibitor of the MBP 85-99 binding to purified HLA-DR2 molecules, and yet, in the antigen presentation assays, it was less effective than YFAK 0.2:0.8. While binding to purified proteins involves interactions of amino acids in the groove of HLA-DR and the anchor residues of the peptide with no contribution from other components, in the whole-cell assay the binding of the antigen to the MHC molecules on the surface of APCs and the interaction with the TCR might be influenced by the actual mode of presentation of the processed epitope to the $\mathrm{T}$ cell. Thus, the lower binding capacity of YFAK 0.2:0.8 might have generated a better $T$ cell epitope.

The $\mathrm{K}$ residues in both YFAK and FEAK are certainly important for the interaction with the TCR, as is the $\mathrm{K}$ at position 93 of MBP 85-99 $(37,38)$. On the other hand, $\mathrm{K}$ located near the $\mathrm{N}$-terminus of the copolymer in the binding site may contribute to stable interactions with the HLA-DR molecules and the TCR, similarly to residue K 307 at P-1 of HA 306-318 bound to HLA-DR1, which can interact with the side chains of $\alpha 1$ helix residues at S $\alpha 53$ or E $\alpha 55$ (54). The A residue in these copolymers functions simply as a spacer for $\mathrm{Y}, \mathrm{F}$, and $\mathrm{K}$.

Of particular importance is the observation that 50mers of YFAK suppressed EAE induced by the PLP 139151 peptide more efficiently than did Cop 1 . Previously, Cop 1 was shown to inhibit EAE induced by either WSCH or the synthetic PLP peptides and to interfere with PLP-specific $T$ cell responses only when mice were coimmunized with both antigens (31), suggesting that copolymers compete for binding to class II MHC molecules. The mechanism of activity of the novel random copolymers might be similar to that of Cop 1 . One possibility is that they lead to the inhibition of binding of the 
potential autoantigenic peptides to class II MHC proteins and subsequent $\mathrm{T}$ cell suppression, although alternatives have also been suggested $(32,33,49)$. Detailed studies of their mechanism(s) - whether by blockade, by induction of $\mathrm{T}$ cell anergy, by induction of regulatory $\mathrm{T}$ cells, or by a combination of these - have been initiated.

Thus, in the present work, several novel random amino acid copolymers were shown to be more potent inhibitors of the autoantigen-specific immune response than Cop 1 both in vitro and in vivo. They appear to be candidates for therapeutic trial in MS.

\section{Acknowledgments}

We thank V.K. Kuchroo for critical review of the manuscript; K.W. Wucherpfennig for cells expressing HLADR2 proteins; J.M. Neveu, R.A. Robinson, and W.S. Lane from the Microchemistry Facility (Harvard University, Cambridge, Massachusetts, USA) for the HPLC separation and sequencing analysis of the copolymers; M.L. Wong for expert technical assistance with mice; and C. Cayetano for the spinal cord tissue processing. This work was supported by grants from the NIH (5R35-CA47554 and AI49524).

Note added in proof. Cop 1 has recently been shown to be more effective in HLA-DR2 (DRB1*1501)-positive than in HLA-DR2-negative MS patients (55).

1. Jersild, C., et al. 1973. Histocompatibility determinants in multiple sclerosis, with special reference to clinical course. Lancet. 2:1221-1225.

2. Spielman, R.S., and Nathenson, N. 1982. The genetics of susceptibility to multiple sclerosis. Epidemiol. Rev. 4:45-65.

3. Olerup, O., and Hillert, J. 1991. HLA class II-associated genetic susceptibility in multiple sclerosis: a critical evaluation. Tissue Antigens. 38:1-15.

4. Zamvil, S.S., et al. 1986. T-cell epitope of the autoantigen myelin basic protein that induces encephalomyelitis. Nature. 324:258-260.

5. Kono, D.H., et al. 1988. Two minor determinants of myelin basic protein induce experimental allergic encephalomyelitis in SJL/J mice. J. Exp. Med. 168:213-227.

6. Madsen, L.S., et al. 1999. A humanized model for multiple sclerosis using HLA-DR2 and human T-cell receptor. Nat. Genet. 23:343-347.

7. Tuohy, V.K., Lu, Z., Sobel, R.A., Laursen, R.A., and Lees, M.B. 1989. Identification of an encephalitogenic determinant of myelin proteolipid protein for SJL mice. J. Immunol. 142:1523-1527.

8. Greer, J.M., Kuchroo, V.K., Sobel, R.A., and Lees, M.B. 1992. Identification and characterization of a second encephalitogenic determinant of myelin proteolipid protein (residues 178-191) for SJL mice. J. Immunol. 149:783-788.

9. Mendel, I., Kerlero de Rosbo, N., and Ben-Nun, A. 1995. A myelin oligodendrocyte glycoprotein peptide induces typical chronic experimental autoimmune encephalomyelitis in $\mathrm{H}-2^{\mathrm{b}}$ mice: fine specificity and $\mathrm{T}$ cell receptor $V$ beta expression of encephalitogenic T cells. Eur. J. Immunol. 25:1951-1959.

10. Santambrogio, L., et al. 1995. Tolerogenic forms of auto-antigens and cytokines in the induction of resistance to experimental allergic encephalomyelitis. J. Neuroimmunol. 58:211-222.

11. Leonard, J.P., Waldburger, K.E., and Goldman, S.J. 1996. Regulation of experimental autoimmune encephalomyelitis by interleukin-12. Ann. NY Acad. Sci. 795:216-226.

12. Gaur, A., Wiers, B., Liu, A., Rothbard, J.B., and Fathman, C.G. 1992. Amelioration of autoimmune encephalomyelitis by myelin basic protein synthetic peptide-induced anergy. Science. 258:1491-1494.

13. Whitacre, C.C., Gienapp, I.E., Meyer, A., Cox, K.L., and Javed, N. 1996. Treatment of autoimmune disease by oral tolerance to autoantigens. Clin. Immunol. Immunopathol. 80:S31-S39.

14. Kennedy, K.J., Smith, W.S., Miller, S.D., and Karpus, W.J. 1997. Induction of antigen-specific tolerance for the treatment of ongoing, relapsing autoimmune encephalomyelitis: a comparison between oral and peripheral tolerance. J. Immunol. 159:1036-1044.

15. Weiner, H.L. 1997. Oral tolerance: immune mechanisms and treatment of autoimmune diseases. Immunol. Today. 18:335-343.
16. Evavold, B.D., and Allen, P.M. 1991. Separation of IL-4 production from Th cell proliferation by an altered $\mathrm{T}$ cell receptor ligand. Science. 252:1308-1310.

17. Karin, N., Mitchell, D.J., Brocke, S., Ling, N., and Steinman, L. 1994. Reversal of experimental autoimmune encephalomyelitis by a soluble peptide variant of a myelin basic protein epitope: $T$ cell receptor antagonism and reduction of interferon gamma and tumor necrosis factor alpha production. J. Exp. Med. 180:2227-2237.

18. Pfeiffer, C., et al. 1995. Altered peptide ligands can control CD4 T lymphocyte differentiation in vivo. J. Exp. Med. 181:1569-1574.

19. Nicholson, L.B., Murtaza, A., Hafler, B.P., Sette, A., and Kuchroo, V.K. 1997. A T cell receptor antagonist peptide induces T cells that mediate bystander suppression and prevent autoimmune encephalomyelitis induced with multiple myelin antigens. Proc. Natl. Acad. Sci. USA. 94:9279-9284.

20. Teitelbaum, D., Meshorer, A., Hirshfeld, T., Arnon, R., and Sela, M. 1971. Suppression of experimental allergic encephalomyelitis by a synthetic polypeptide. Eur. J. Immunol. 1:242-248.

21. McDevitt, H.O., and Sela, M. 1965. Genetic control of the antibody response. I. Demonstration of determinant-specific differences in response to synthetic polypeptide antigens in two strains of inbred mice. J. Exp. Med. 122:517-532.

22. McDevitt, H.O., and Sela, M. 1967. Genetic control of the antibody response. II. Further analysis of the specificity of determinant-specific control, and genetic analysis of the response to $(\mathrm{H}, \mathrm{G})-\mathrm{A}-\mathrm{L}$ in CBA and C57 mice. J. Exp. Med. 126:969-978.

23. Teitelbaum, D., Webb, C., Meshorer, A., Arnon, R., and Sela, M. 1973. Suppression by several polypeptides of experimental allergic encephalomyelitis induced in guinea pigs and rabbits with bovine and human basic encephalitogen. Eur. J. Immunol. 3:273-279.

24. Teitelbaum, D., et al. 1974. Suppression of experimental allergic encephalomyelitis in rhesus monkeys by a synthetic basic copolymer. Clin. Immunol. Immunopathol. 3:256-262.

25. Aharoni, R., Teitelbaum, D., and Arnon, R. 1993. T suppressor hybridomas and interleukin-2-dependent lines induced by copolymer 1 or by spinal cord homogenate down-regulate experimental allergic encephalomyelitis. Eur. J. Immunol. 23:17-25.

26. Bornstein, M.B., et al. 1987. A pilot trial of copolymer 1 in exacerbatingremitting multiple sclerosis. N. Engl. J. Med. 317:408-414.

27. Johnson, K.P., et al. 1995. Copolymer 1 reduces relapse rate and improves disability in relapsing-remitting multiple sclerosis: results of a phase III multicenter, double-blind placebo-controlled trial. The Copolymer 1 Multiple Sclerosis Study Group. Neurology. 45:1268-1276.

28. Johnson, K.P., et al. 1998. Extended use of glatiramer acetate (Copaxone) is well tolerated and maintains its clinical effect on multiple sclerosis relapse rate and degree of disability. Copolymer 1 Multiple Sclerosis Study Group. Neurology. 50:701-708.

29. Fridkis-Hareli, M., et al. 1994. Direct binding of myelin basic protein and synthetic copolymer 1 to class II major histocompatibility complex molecules on living antigen-presenting cells: specificity and promiscuity. Proc. Natl. Acad. Sci. USA. 91:4872-4876.

30. Ben-Nun, A., et al. 1996. The autoimmune reactivity to myelin oligodendrocyte glycoprotein (MOG) in multiple sclerosis is potentially pathogenic: effect of copolymer 1 on MOG-induced disease. J. Neurol. 243:S14-S22.

31. Teitelbaum, D., Fridkis-Hareli, M., Arnon, R., and Sela, M. 1996. Copolymer 1 inhibits chronic relapsing experimental allergic encephalomyelitis induced by proteolipid protein (PLP) peptides in mice and interferes with PLP-specific T cell responses. J. Neuroimmunol. 64:209-217.

32. Duda, P.W., Schmied, M.C., Cook, S.L., Krieger, J.I., and Hafler, D.A. 2000. Glatiramer acetate (Copaxone) induces degenerate, Th2-polarized immune responses in patients with multiple sclerosis. J. Clin. Invest. 105:967-976.

33. Duda, P.W., Krieger, J.I., Schmied, M.C., Balentine, C., and Hafler, D.A. 2000. Human and murine CD4 T cell reactivity to a complex antigen: recognition of the synthetic random polypeptide glatiramer acetate. J. Immunol. 165:7300-7307.

34. Fridkis-Hareli, M., Stern, J.N.H., Fugger, L., and Strominger, J.L. 2001. Synthetic peptides that inhibit binding of the myelin basic protein 85-99 epitope to multiple sclerosis-associated HLA-DR2 molecules and MBP-specific T-cell responses. Hum. Immunol. 62:753-763.

35. Fridkis-Hareli, M., and Strominger, J.L. 1998. Promiscuous binding of synthetic copolymer 1 to purified HLA-DR molecules. J. Immunol. 160:4386-4397.

36. Krieger, J.I., et al. 1991. Single amino acid changes in DR and antigen define residues critical for peptide-MHC binding and $\mathrm{T}$ cell recognition. J. Immunol. 146:2331-2340.

37. Wucherpfennig, K.W., et al. 1994. Structural requirements for binding of an immunodominant myelin basic protein peptide to DR2 isotypes and for its recognition by human T cell clones. J. Exp. Med. 179:279-290.

38. Smith, K.J., Pyrdol, J., Gauthier, L., Wiley, D.C., and Wucherpfennig, K.W. 1998. Crystal structure of HLA-DR2 (DRA*0101, DRB1*1501) com- 
plexed with a peptide from human myelin basic protein. J. Exp. Med. 19:1511-1520.

39. Kalandadze, A., Galleno, M., Foncerrada, L., Strominger, J.L., and Wucherpfennig, K.W. 1996. Expression of recombinant HLA-DR2 molecules. J. Biol. Chem. 271:20156-20162.

40. Falk, K., et al. 2000. Induction and suppression of an autoimmune disease by oligomerized $\mathrm{T}$ cell epitopes: enhanced in vivo potency of encephalitogenic peptides. J. Exp. Med. 191:717-730.

41. Santambrogio, L., et al. 1993. Studies on the mechanisms by which transforming growth factor-beta (TGF-beta) protects against allergic encephalomyelitis. Antagonism between TGF-beta and tumor necrosis factor. J. Immunol. 151:1116-1127.

42. Krogsgaard, M., et al. 2000. Visualization of myelin basic protein T-cell epitopes in multiple sclerosis lesions using a monoclonal antibody specific for the HLA-DR2-MBP 85-99 complex. J. Exp. Med. 191:1395-1412.

43. Vandenbark, A., Hashim, A.G., and Offner, H. 1989. Immunization with a synthetic $\mathrm{T}$-cell receptor $\mathrm{V}$-region peptide against experimental autoimmune encephalomyelitis. Nature. 341:541-544.

44. Wraith, D.C., McDevitt, H.O., Steinman, L., and Acha-Orbea, H. 1989. T cell recognition as the target for immune intervention in autoimmune disease. Cell. 57:709-715.

45. Hohlfeld, R. 1997. Biotechnological agents of the immunotherapy of multiple sclerosis. Principles, problems and perspectives. Brain. 120:865-916.

46. Brocke, S., et al. 1996. Treatment of experimental encephalomyelitis with a peptide analogue of myelin basic protein [erratum 1998, 392:630] Nature. 379:343-346.

47. Bielekova, B., et al. 2000. Encephalitogenic potential of the myelin basic protein peptide (amino acids 83-99) in multiple sclerosis: results of a phase II clinical trial with an altered peptide ligand. Nat. Med. 10:1167-1175.

48. Kappos, L., et al. 2000. Induction of a non-encephalitogenic type $2 \mathrm{~T}$ helper-cell autoimmune response in multiple sclerosis after administration of an altered peptide ligand in a placebo-controlled, randomized phase II trial. Nat. Med. 10:1176-1182.

49. Aharoni, R., Teitelbaum, D., Arnon, R., and Sela, M. 1999. Copolymer 1 acts against the immunodominant epitope 82-100 of myelin basic protein by $\mathrm{T}$ cell receptor antagonism in addition to major histocompatibility complex blocking. Proc. Natl. Acad. Sci. USA. 96:634-639.

50. Johnson, K.P., et al. 2000. Sustained clinical benefits of glatiramer acetate in relapsing multiple sclerosis patients observed for 6 years. Mult. Scler. 6:255-266.

51. Fridkis-Hareli, M., Teitelbaum, D., Arnon, R., and Sela, M. 1995. Synthetic copolymer 1 and myelin basic protein do not require processing prior to binding to class II major histocompatibility complex molecules on living antigen-presenting cells. Cell. Immunol. 163:229-236.

52. Fridkis-Hareli, M., Teitelbaum, D., Pecht, I., Arnon, R., and Sela, M. 1997. Binding of copolymer 1 and myelin basic protein leads to clustering of class II MHC molecules on antigen-presenting cells. Int. Immunol. 9:925-934.

53. Rotzschke, O., Falk, K., and Strominger, J.L. 1997. Superactivation of an immune response triggered by oligomerized T cell epitopes. Proc. Natl. Acad. Sci. USA. 94:14642-14647.

54. Stern, L.J., et al. 1994. Crystal structure of the human class II MHC protein HLA-DR1 complexed with an influenza virus peptide. Nature. 368:215-221.

55. Fusco, C., et al. 2001. HLA-DRB1*1501 and response to copolymer-1 therapy in relapsing-remitting multiple sclerosis. Neurology. 57:1976-1979. 